\title{
Efecto del tipo de forraje y la inclusión de quebracho en la dieta de ovejas lactantes sobre sus rendimientos productivos
}

\author{
S. Lobón, A. Sanz, M. Blanco y M. Joy* \\ Centro de Investigación y Tecnología Agroalimentaria de Aragón (CITA). Instituto Agroalimentario de \\ Aragón - IA2 (CITA-Universidad de Zaragoza). Avda. Montañana, 930, 50059, Zaragoza, España
}

\begin{abstract}
Resumen
Se estudió el efecto del tipo de forraje y el uso de quebracho en la dieta de ovejas sobre sus rendimientos y los de sus corderos lechales. Para ello, se utilizaron 39 ovejas con sus corderos distribuidos en 4 lotes según un diseño factorial de $2 \times 2$, con dos tipos de forraje (Pastoreo de pradera vs. Heno de pradera) y dos tipos de concentrado [concentrado comercial (Control) vs. concentrado con quebracho (QUE)]. El ensayo se prolongó desde el nacimiento hasta el sacrificio de los corderos a un peso vivo de $10-12 \mathrm{~kg}$. La producción de leche y su contenido proteico fueron superiores en las ovejas Pastoreo que en las de Heno, con un contenido de grasa similar. Las ovejas de Pastoreo presentaron mayor concentración plasmática de urea y proteína total, y menor de ácidos grasos no esterificados. Los corderos de Pastoreo fueron más pesados al sacrificio y presentaron mayor rendimiento canal que los corderos de Heno a la misma edad. La inclusión de quebracho en el concentrado de ovejas no afectó al peso, producción lechera o concentración de metabolitos plasmáticos de las ovejas; únicamente redujo el contenido proteico de la leche en un $4 \%$ y aumentó el peso vivo del cordero en la tercera y cuarta semana de lactación, que tendió a alcanzar el peso de sacrificio 3,5 días antes que el resto de corderos. En nuestras condiciones de trabajo, las ovejas lactantes en pastoreo y sus corderos lechales obtuvieron mejores rendimientos que las ovejas estabuladas con heno. La inclusión de quebracho no presentó efectos consistentes.
\end{abstract}

Palabras clave: Pastoreo, taninos condensados, leche, calidad de canal, cordero lechal.

\begin{abstract}
Effect of forage type and the inclusion of quebracho in the diet of lactating ewes on their performances

The effects of the type of forage and the inclusion of quebracho in the diet of lactating ewes on the performance of the ewes and their suckling lambs were assessed. Ewe-lamb pairs $(n=39)$ were assigned in 4 lots according to a factorial design $2 \times 2$, with two types of forage (Pasture vs. Hay) and two types of concentrates [commercial concentrate (Control) vs. concentrate with quebracho (QUE)]. The experimental period lasted from lambing until the lambs reached 10-12 kg body weight. Regarding the type of forage, milk yield and protein content were greater in Pasture than in Hay ewes, with similar fat content. Pasture ewes presented greater plasma urea and total protein and lower non esterified fatty acids concentrations than Hay ewes. Pasture suckling lambs were heavier at slaughter than Hay lambs at the same age. The inclusion of quebracho in the ewe s concentrate did not affect the ewe performances or plasma metabolite concentrations, only reduced milk protein content by $4 \%$. Lamb's body weight increased at third and four week of lactation with the inclusion of quebracho, tending to reach slaughter weight at a slightly younger age. In our work conditions, Pasture lactating ewes and their suckling lambs obtained better performance than Hay indoors ewes. The inclusion of quebracho did not show consistent effects.
\end{abstract}

Keywords: Grazing, condensed tannins, milk, carcass quality, suckling lamb.

\footnotetext{
* Autor para correspondencia: mjoy@aragon.es http://doi.org/10.12706/itea.2017.023
} 


\section{Introducción}

En zonas del mediterráneo, especialmente en España, la producción de cordero mayoritaria está basada en corderos ligeros sacrificados con un peso vivo (PV) de 18-26 kg. Sin embargo, también se comercializan corderos lechales de diferentes razas. Dichos corderos lechales se alimentan exclusivamente de leche materna desde el nacimiento hasta el sacrificio, que se realiza a los 35-45 días de edad y 10-12 kg de PV (Sanz et al., 2008). En estos sistemas, las ovejas suelen estabularse alrededor del parto, se alimentan con heno y se suplementan con concentrado para cubrir los mayores requerimientos nutricionales de la lactación (Ripoll-Bosch et al., 2014). No obstante, cuando hay pasto disponible, las ovejas junto con sus corderos pueden pastar sin encontrarse efectos negativos en los rendimientos de ambos (Joy et al., 2012). Este sistema de alimentación es una interesante alternativa a la estabulación ya que utiliza recursos naturales, cumpliendo con las demandas de los consumidores que consideran los sistemas de pastoreo más sostenibles y con mayor respeto al bienestar animal (Montossi et al., 2013).

En los últimos años ha crecido el interés sobre la inclusión de compuestos secundarios en las dietas de rumiantes, debido principalmente al hecho de que reducen las emisiones de metano, y además pueden mejorar los rendimientos productivos de los animales, aunque con resultados variables dependiendo del tipo de compuesto y la dosis ingerida (Min et al., 2003; Waghorn, 2008; Patra y Saxena, 2011). Concretamente, se ha estudiado el efecto de la inclusión de taninos condensados (TC) sobre los rendimientos de los animales, aunque no se conoce bien el impacto específico ni en que vías metabólicas participan. Además, los estudios en sistemas de producción de corderos lechales son escasos, por lo que sería interesante profundizar más en ellos. El quebracho (Schinopsis balansae) se ha utili- zado como fuente de TC en diferentes ensayos con animales, incorporado en el concentrado. Para estudiar el efecto del quebracho en la producción de lechales, dado que el cordero lechal únicamente ingiere leche de la oveja, el quebracho debería ser incorporado en el concentrado de las madres. Se debería estudiar el efecto directo del quebracho sobre los rendimientos de las ovejas y el efecto indirecto sobre el cordero, por la posible transferencia de polifenoles a la carne del cordero. Moñino et al. (2008) confirmaron la transferencia de polifenoles a la carne del cordero procedentes de la dieta de su madre. El objetivo de este estudio fue evaluar el efecto del tipo de forraje (Pastoreo vs. Heno) y la inclusión de quebracho en la dieta de ovejas lactantes (Control vs. QUE) sobre sus rendimientos y los de sus corderos lechales.

\section{Material y métodos}

El comité ético del CITA de Aragón aprobó los procedimientos experimentales y de sacrificio utilizados en este estudio, que están de acuerdo con la directiva europea 2010/63 de protección de animales para el uso experimental y para otros propósitos específicos.

\section{Animales y diseño experimental}

El experimento se llevó a cabo en La Estación Experimental de la Garcipollera, situada en el Pirineo $\left(42^{\circ} 37^{\prime} \mathrm{N}, 0^{\circ} 30^{\prime} \mathrm{O}, 945 \mathrm{~m}\right.$ sobre el nivel del mar), durante la primavera del año 2014. El ensayo comenzó el 18 de marzo y finalizó el 6 de mayo. La temperatura media durante este periodo fue de $8,28,12,47$ y $12,45^{\circ} \mathrm{C}$ en marzo, abril y mayo, con una precipitación de 49,5, 107,4 y 0,16 mm respectivamente, concentrándose las lluvias los días $23,24,26$ y 30 de marzo, 2, 3, 5, 10, 12, 13, $19,20,21,23$ y 24 de abril y 3 de mayo. 
Después del parto, se seleccionaron 39 ovejas de raza Churra Tensina de parto simple y sus corderos, y se distribuyeron en uno de los cuatro tratamientos siguiendo un diseño experimental de $2 \times 2$. La distribución se realizó teniendo en cuenta la fecha de parto (18/03/ $2014 \pm 2,4)$, el PV $(46,6 \pm 3,9)$, la condición corporal (CC) $(2,43 \pm 0,16)$ y la edad de la oveja $(6,8 \pm 2,2)$, así como el sexo del cordero.

Los factores estudiados fueron el tipo de forraje (Pastoreo de pradera vs. Heno de pradera en estabulación) y la inclusión de quebracho en el concentrado de las ovejas [concentrado comercial (Control) vs. concentrado con $10 \%$ de quebracho (QUE, SYLVAFEED ByPro Q, Adial Nutricion. Gerona, España, con $75 \%$ de TC)]. Todas las ovejas recibieron $300 \mathrm{~g} / \mathrm{d}$ de concentrado a las 8:00 de la mañana. En cada tratamiento de forraje, las ovejas se dividieron en dos grupos, que recibieron diariamente concentrado QUE $(n=18)$ o concentrado Control $(n=21)$. Todos los animales tuvieron acceso al agua y bloques minerales durante el experimento.

El pastoreo se realizó en dos parcelas de pradera permanente de valle de montaña, de 0,5 ha cada una. En cada parcela se mantuvo un máximo de 10 ovejas con sus crías. El heno se ofreció ad libitum a las ovejas estabuladas, y se obtuvo de las mismas parcelas en la primavera del año anterior. Los ingredientes principales del concentrado comercial fueron: cebada $(33,5 \%)$, salvado $(20 \%)$, gluten feed de maíz $(18 \%)$, harina de girasol (10\%) y maíz (10\%); y los del concentrado QUE fueron: cebada (48,7\%), gluten feed de maíz (15\%), harina de soja $(12,1 \%)$, quebracho $(10 \%)$, salvado $(6,0 \%)$ y maíz $(5 \%)$. Los corderos se criaron con sus madres hasta que alcanzaron el PV de la categoría lechal (10-12 $\mathrm{kg}$ PV). Una vez alcanzaron el PV objetivo, los lechales se trasladaron al matadero experimental del CITA en Zaragoza $(180 \mathrm{~km})$ para proceder a su sacrificio.

\section{Muestreos y controles}

Todos los muestreos y controles se realizaron semanalmente. La producción forrajera se estimó mediante siega manual a $3 \mathrm{~cm}$ del suelo de 10 cuadros de $0,25 \mathrm{~m}^{2}$ por ha. También se determinó la altura de la hierba tomando 100 puntos aleatorios por hectárea. Se tomaron muestras de la dieta ofrecida (heno, pradera y concentrado), que se desecaron en estufa de ventilación forzada a $60^{\circ} \mathrm{C}$ hasta peso constante. Posteriormente se molieron y se almacenaron hasta su análisis químico.

Los animales se pesaron, previamente a la oferta de concentrado, con una balanza electrónica $(0,1 \mathrm{~kg}$ precisión). La ganancia media diaria (GMD) de los corderos se estimó a partir de la regresión lineal del PV con respecto al tiempo. Además, dos técnicos entrenados estimaron la CC de las ovejas siguiendo la metodología propuesta por Russel et al. (1969).

Se estimó la producción de leche siguiendo la metodología de Donney et al. (1979), con administración de 5 UI de oxitocina en la vena yugular previamente al ordeño a máquina y con acabado manual (intervalo entre ordeños, 4 horas). En cada ordeño, se tomó una muestra de leche por animal, con dicromato potásico para su conservación y mantenida a $4^{\circ} \mathrm{C}$ hasta su posterior análisis químico. La producción de leche estándar se calculó como: Producción de leche estándar $(\mathrm{l} / \mathrm{d})=$ producción de leche $(\mathrm{I} / \mathrm{d}) \times[(0,0071 \times$ grasa bruta $(\mathrm{g} / \mathrm{l})+(0,0043 \mathrm{x}$ proteína bruta $(g / l))+0,2224]$ (Bocquier et al., 1993).

Se tomaron muestras de sangre de la vena yugular en tubos de vacío con heparina (Vacuette, España) para el análisis de metabolitos en sangre (proteínas totales, ácidos grasos no esterificados (AGNE) y urea). Las muestras se centrifugaron a $3000 \times g$ durante 15 min y a $4^{\circ} \mathrm{C}$, y se congeló el plasma obtenido a $-20^{\circ} \mathrm{C}$ hasta su análisis posterior. 
Los corderos se pesaron antes y después de su traslado al matadero experimental, para estimar las pérdidas por transporte

$\left(\frac{P V_{\text {pre-transporte }}-P V_{\text {post-transporte }}}{P V_{\text {pre-transporte }}} \times 100\right)$. Tras el sacri-

ficio, las canales se pesaron para obtener el peso canal caliente (PCC), se almacenaron a $4^{\circ} \mathrm{C}$ durante 24 horas, y se volvieron a pesar para obtener el peso canal fría (PCF). A partir de estos datos se calcularon el rendimiento al matadero $\left(\frac{P C F}{P V_{\text {sacrificio }}} \times 100\right)$ y las pérdidas por refrigeración $\left(\frac{P C C-P C F}{P C C} \times 100\right)$.

\section{Análisis químicos}

\section{Alimentos}

Los contenidos de materia seca (MS) y en cenizas se determinaron según los métodos del AOAC (1999). Los contenidos de fibra neutro detergente (FND), fibra ácido detergente (FAD) y lignina ácido detergente (LAD) se determinaron siguiendo el procedimiento secuencial de Van Soest et al. (1991), adaptado al analizador de fibra Ankom200/220 (Ankom, NY, EE. UU.). Todos los valores obtenidos para las fibras se corrigieron por el contenido en cenizas, mostrando los resultados libres de cenizas. El contenido en proteína bruta (PB) (Nitrógeno x 6,25) se determinó mediante el procedimiento de Dumas (AOAC, 1999), usando un analizador de nitrógeno (Modelo NA 2100, CE Instruments, Thermoquest SA, Barcelona, España). La energía metabolizable de los ingredientes de la dieta se estimó usando la ecuación propuesta por Mertens (1983). Los taninos condensados (TC) totales se determinaron con el método colorimétrico del $\mathrm{HCL}$-butanol descrito por Grabber et al. (2013).

\section{Leche}

Las muestras de leche se analizaron en un Combifoss 5000 device (Foss, Hillerød, Dinamarca) que comprende un Fossomatic 5000, contador de células somáticas, y un MilkoScan 4000, para determinar proteína bruta y grasa bruta. Los análisis se realizaron en el Laboratorio Interprofesional Lechero de Aragón.

\section{Plasma}

Las concentraciones en plasma de urea y proteína total (métodos enzimáticos-colorimétricos) se determinaron con un analizador automático (Atom A-25, Byosistems, Barcelona, España). El contenido de AGNE en el plasma se determinó usando un analizador automático (GernonStar; RAL/TRANSASIA, Dabhel, India) utilizando reactivos de Randox (Randox Laboratories, Crumlin, Antrim, Reino Unido). Las referencias comerciales de las muestras de plasma (suero bovino precisión, Randox Laboratories) se usaron para evaluar la fiabilidad del análisis.

\section{Análisis estadísticos}

Los datos se analizaron usando el software estadístico SAS (SAS V.9.3). El PV, la CC y los metabolitos en plasma de la oveja y el PV del cordero se analizaron mediante el modelo mixto de medidas repetidas con el tipo de forraje, la inclusión de quebracho en el concentrado, la semana de la lactación y sus interacciones como efectos fijos y oveja/cordero como efecto aleatorio. Se ajustaron los grados de libertad con la corrección de Keward-Roger para tener en cuenta el número no equilibrado de muestras por tratamiento o la ausencia de algún dato. La producción de leche y su composición se analizaron con el mismo modelo pero además se incluyó el PV de la oveja como covariable. Se probaron di- 
ferentes estructuras de la matriz de varianzas y covarianzas en cada modelo para especificar las relaciones entre los errores y se eligió la que menor criterio de información Aikake presentó. La ganancia de peso del cordero, las pérdidas de peso, la edad al sacrificio y los parámetros de la canal se analizaron mediante análisis de varianza (ANOVA) con un modelo lineal general (GLM) con el tipo de forraje, la inclusión de quebracho y su interacción como efectos fijos. Se calcularon las medias mínimo cuadráticas y la comparación entre medias se hizo con el test de Tukey. Las interacciones o covariables no significativas se quitaron del modelo y se repitió el análisis. El nivel de significación se fijó en $\mathrm{P}<0,05$ y se discutieron las tendencias cuando $0,05 \leq \mathrm{P}<0,10$.

\section{Resultados}

\section{Alimentos}

El estudio comenzó el 18 de marzo de 2014, cuando el forraje iniciaba su crecimiento. La altura del forraje y la producción forrajera varió entre 3,5 y $8 \mathrm{~cm}$, y 50 y $1.200 \mathrm{~kg} \mathrm{MS} / \mathrm{ha}$, respectivamente, siendo la producción media de $437 \pm 113 \mathrm{~kg} \mathrm{MS} / \mathrm{ha}$. En la Tabla 1 se muestra la composición química de los alimentos ofrecidos a las ovejas. La pradera fresca presentó un alto contenido de $\mathrm{PB}$, que fue casi 3,5 veces mayor que el observado en el heno de pradera, y un bajo contenido de FND, $30 \%$ inferior al del heno. Por lo tanto, el valor estimado de la energía metabolizable

Tabla 1. Composición química de los alimentos utilizados en la dieta de las ovejas en lactación Table 1. Chemical composition of the feedstuffs used during the ewe lactation period

\begin{tabular}{lrrrr}
\hline & \multicolumn{2}{c}{ Forraje } & \multicolumn{2}{c}{ Concentrado } \\
\cline { 2 - 5 } & Heno & Pastoreo & QUE $^{1}$ & Control \\
\hline Humedad, g/kg & 110,5 & 828,1 & 110,1 & 113,6 \\
Cenizas, g/kg MS & 78,0 & 118,6 & 62,2 & 62,6 \\
Proteína bruta, g/kg MS & 69,2 & 239,3 & 140,6 & 140,0 \\
Fibra Neutro Detergente, g/kg MS & 632,8 & 446,3 & 175,3 & 248,8 \\
Fibra Ácido Detergente, g/kg MS & 338,5 & 185,4 & 59,8 & 69,4 \\
Lignina Ácido Detergente, g/kg MS & 40,1 & 39,5 & 10,9 & 20,8 \\
Energía metabolizable estimada, MJ/ kg MS & 8,2 & 11,6 & 16,1 & 15,1 \\
TC2 totales, g cyanidina/kg MS & 2,1 & 3,2 & 8,1 & 1,0 \\
\hline
\end{tabular}

${ }^{1}$ QUE: $10 \%$ de quebracho; ${ }^{2}$ Taninos Condensados.

(EM) fue $29,3 \%$ mayor para la pradera que para el heno. Respecto a los concentrados, presentaron un contenido de proteína e energía similar, mientras que el contenido en fibras y TC difirió. El concentrado QUE presentó sobre todo menor FND y LAD y mayor cantidad de TC.

\section{Parámetros productivos de las ovejas}

No se observaron interacciones significativas entre el tipo de forraje y la inclusión de quebracho en el concentrado $(P>0,05)$. Por ello, los resultados se presentan por separado para estos dos efectos principales. 
Las ovejas partieron de PV y CC similares al parto (Tabla 2). Sin embargo, el tipo de forraje tendió a afectar a la evolución de PV durante la lactación $(P<0,10)$ y afectó a la evolución de la CC durante la lactación $(P<0,001)$. Durante la lactación, las ovejas del lote Pastoreo mantuvieron el PV y la CC, mientras que las ovejas del lote Heno presentaron pérdidas en ambos parámetros. La inclusión de quebracho en el concentrado no tuvo efecto ni en el PV ni en la CC de las ovejas $(P>0,05)$.
La producción y calidad de la leche se muestran en la Tabla 3. La producción de leche se vio afectada por la interacción entre el tipo de forraje y la semana de lactación $(\mathrm{P}<0,05$; Figura 1). Las ovejas de Pastoreo y de Heno presentaron similar producción de leche durante las 2 primeras semanas de lactación, pero entre la $3^{\mathrm{a}}$ y $5^{\mathrm{a}}$ semana fue mayor en las ovejas en Pastoreo $(P<0,05)$. Las ovejas de Pastoreo presentaron una producción de leche creciente con 1,1 vs. 1,5 l/d, primera vs. quinta se-

Tabla 2. Efecto del tipo de forraje (F) y la inclusión de quebracho en el concentrado (C) de ovejas en lactación sobre sus parámetros productivos

Table 2. Effect of the type of forage (F) and the inclusion of quebracho in the concentrate (C) of lactating ewes on their productive performance

\begin{tabular}{|c|c|c|c|c|c|c|c|}
\hline & \multicolumn{2}{|c|}{ Forraje } & \multicolumn{2}{|c|}{ Concentrado } & \multirow[b]{2}{*}{$\mathrm{EE}^{2}$} & \multicolumn{2}{|c|}{ Efecto $^{3}$} \\
\hline & Heno & Pastoreo & QUE $^{1}$ & Control & & $\mathrm{F}$ & $\mathrm{C}$ \\
\hline$n$ & 19 & 20 & 18 & 21 & & - & - \\
\hline \multicolumn{8}{|l|}{ Al parto } \\
\hline Peso vivo, kg & 47,9 & 45,3 & 46,6 & 46,6 & 3,9 & NS & NS \\
\hline Condición corporal & 2,45 & 2,41 & 2,41 & 2,45 & 0,16 & NS & NS \\
\hline \multicolumn{8}{|c|}{ Variación durante la lactación } \\
\hline Peso vivo, kg & $-1,8$ & 0,3 & $-1,2$ & $-0,3$ & 2,1 & $\dagger$ & NS \\
\hline Condición corporal & $-0,29$ & 0 & $-0,11$ & $-0,18$ & 0,13 & $* * *$ & NS \\
\hline
\end{tabular}

${ }^{1}$ QUE: $10 \%$ de quebracho; ${ }^{2}$ Error estándar; ${ }^{3} \dagger=\mathrm{P}<0,10$.

mana de lactación $(P<0,05)$. En cambio, las ovejas de Heno tuvieron una producción creciente hasta la segunda semana, manteniéndose constante durante las dos siguientes semanas y disminuyendo en la quinta semana.

El contenido en PB de la leche también se vio afectado por la interacción entre el tipo de forraje y la semana de lactación (Tabla 3). La evolución del contenido en PB siguió un patrón similar a la producción de leche, con mayor contenido en el tratamiento de Pastoreo que en el de Heno durante las 3 últi- mas semanas de lactación ( $P<0,05$; Figura 1 ). La producción de leche estándar se vio afectada únicamente por el tipo de forraje (Tabla $3)$, siendo un $21 \%$ superior en el tratamiento de Pastoreo $(P<0,001)$. La inclusión de quebracho tendió a incrementar la producción estándar de leche $(P<0,10)$ y redujo el contenido de proteína $(P<0,05)$ (Figura 1).

La semana de lactación afectó a todos los parámetros de leche estudiados $(P<0,05)$ excepto a la producción de leche estándar $(P>0,05 ;$ Tabla 3). Durante la primera semana 
Tabla 3. Efecto del tipo de forraje (F), la inclusión de quebracho en el concentrado (C) y la semana de lactación (S) sobre la producción y calidad de la leche de las ovejas lactantes Table 3. Effect of the type of forage (F), the inclusion of quebracho in the concentrate (C) and the week of lactation (W) on milk yield and quality of lactating ewes

\begin{tabular}{|c|c|c|c|c|c|c|c|c|}
\hline & \multicolumn{2}{|c|}{ Forraje } & \multicolumn{2}{|c|}{ Concentrado } & \multirow[b]{2}{*}{$\mathrm{EE}^{2}$} & \multicolumn{3}{|c|}{ Efecto $^{3}$} \\
\hline & Heno & Pastoreo & QUE$^{1}$ & Control & & $\mathrm{F}$ & C & $\mathrm{S}$ \\
\hline Producción de leche, l/d 4 & 1,01 & 1,32 & 1,22 & 1,12 & 0,04 & $* * *$ & NS & * \\
\hline Producción de leche estándar, l/d & 0,88 & 1,12 & 1,08 & 0,92 & 0,04 & $* * *$ & $\dagger$ & NS \\
\hline Grasa bruta, \% & 6,50 & 6,35 & 6,57 & 6,29 & 0,19 & NS & NS & $* * *$ \\
\hline Proteína bruta, \% & 4,68 & 5,12 & 4,78 & 5,01 & 0,07 & $* * *$ & * & * \\
\hline Lactosa, \% & 5,06 & 5,08 & 5,06 & 5,08 & 0,03 & NS & NS & *** \\
\hline Log_SCC 5 & 2,41 & 2,35 & 2,28 & 2,48 & 0,09 & NS & NS & * \\
\hline
\end{tabular}

${ }^{1}$ QUE: $10 \%$ de quebracho; ${ }^{2}$ Error estándar; ${ }^{3} \dagger=\mathrm{P}<0,10 ;{ }^{4}$ Interacción significativa entre el tipo de forraje y la semana de lactación (Ver Figura 1); ${ }^{5}$ Número de células somáticas.

de lactación, la leche presentó un mayor contenido de grasa, proteína y número de células somáticas y menor contenido de lactosa que las restantes semanas $(P<0,05)$, que presentaron contenidos similares $(P>0,05$; datos no mostrados).

La evolución de los metabolitos plasmáticos de las ovejas se muestra en la Figura 2. El tipo de forraje $(P<0,05)$ y la semana de lactación $(P<0,01)$ afectaron a la concentración de proteína total del plasma sin presentar interacción entre ambos ( $P>0,05)$. En promedio, la concentración de proteína total fue mayor en las ovejas en Pastoreo que en las ovejas Heno $(66,1$ vs. $63,1 \mathrm{~g} / \mathrm{l} ; \mathrm{P}<0,05)$. Respecto a la semana de lactación, la concentración de proteína total fue mayor en la primera semana de lactación $(P<0,05)$, disminuyendo progresivamente hasta la quinta semana, momento en que la concentración de proteína total fue similar al resto de las semanas $(P<0,05)$. La concentración de AGNE en plasma presentó una interacción entre el tipo de fo- rraje y la semana de lactación $(P<0,05)$. Las concentraciones fueron similares durante las dos primeras semanas de lactación $(P>0,05)$, posteriormente las ovejas del tratamiento Heno presentaron mayor concentración que las del tratamiento Pastoreo $(P<0,05)$. La concentración de urea en plasma también presentó una interacción entre el forraje y la semana de lactación $(P<0,05)$. La concentración de urea plasmática siempre fue mayor en ovejas en Pastoreo que en ovejas con Heno $(P<0,05)$, incrementándose la diferencia entre tratamientos a medida que la lactación avanzaba. Las ovejas del tratamiento Heno presentaron un concentración de urea constante, mientras que en las del Pastoreo aumentó hasta la tercera semana de lactación $(13,18$ $\mathrm{mmol} / \mathrm{l})$, manteniéndose constante posteriormente $(P<0,05)$.

La inclusión de quebracho en el concentrado no afectó la concentración plasmática de ninguno de los metabolitos estudiados ( $P>0,05$; Figura 2). 


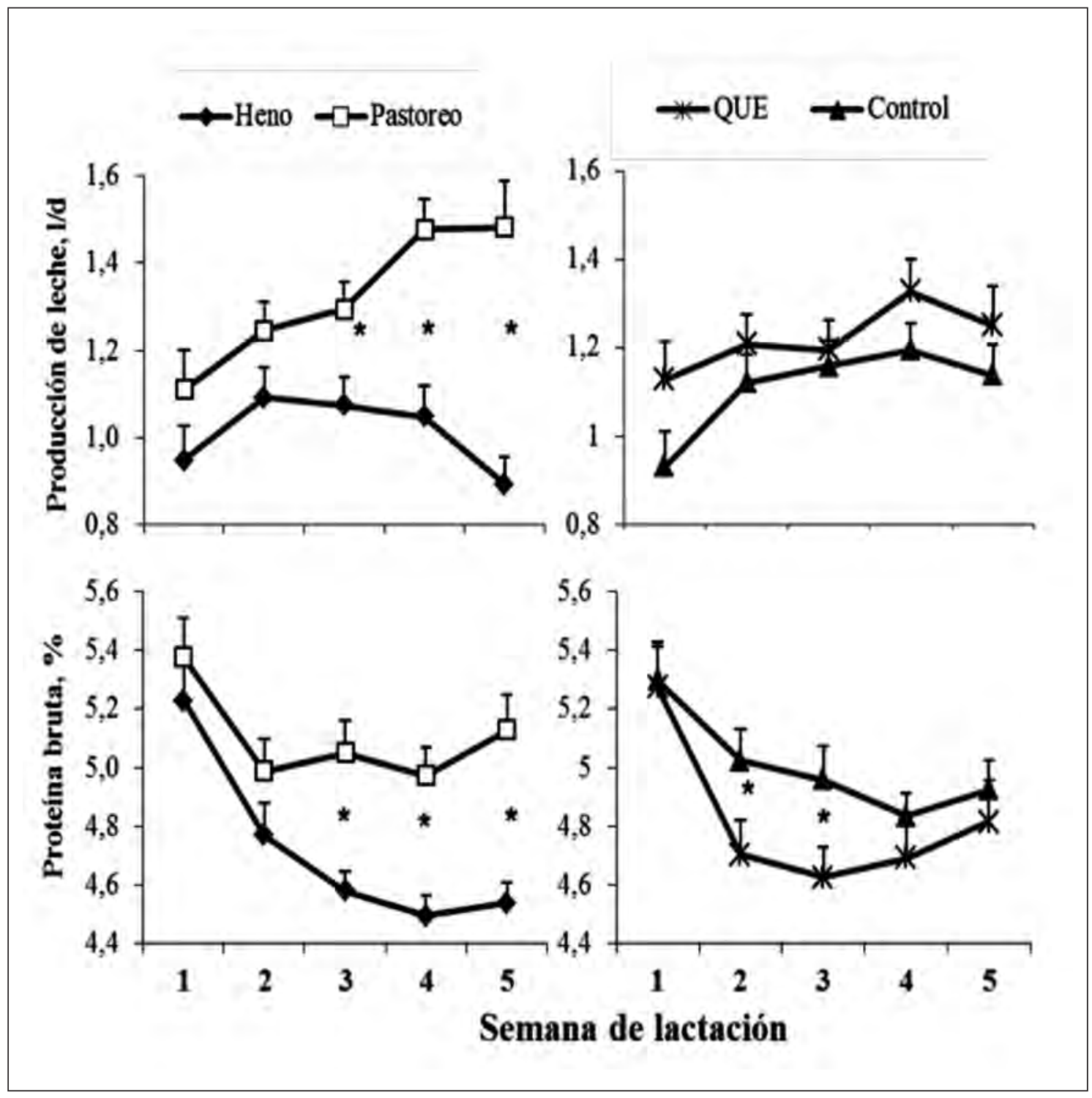

Figura 1. Efecto del tipo de forraje (Heno vs. Pastoreo) y la inclusión de quebracho en el concentrado (QUE vs. Control) de las dietas de ovejas sobre la producción de leche y el contenido en proteína bruta a lo largo de la lactación.

Figure 1. Effect of the forage type (Pasture vs. Hay) and the inclusion of quebracho in the concentrate (QUE vs. Control) of lactating ewes on milk yield and crude protein content during lactation. 


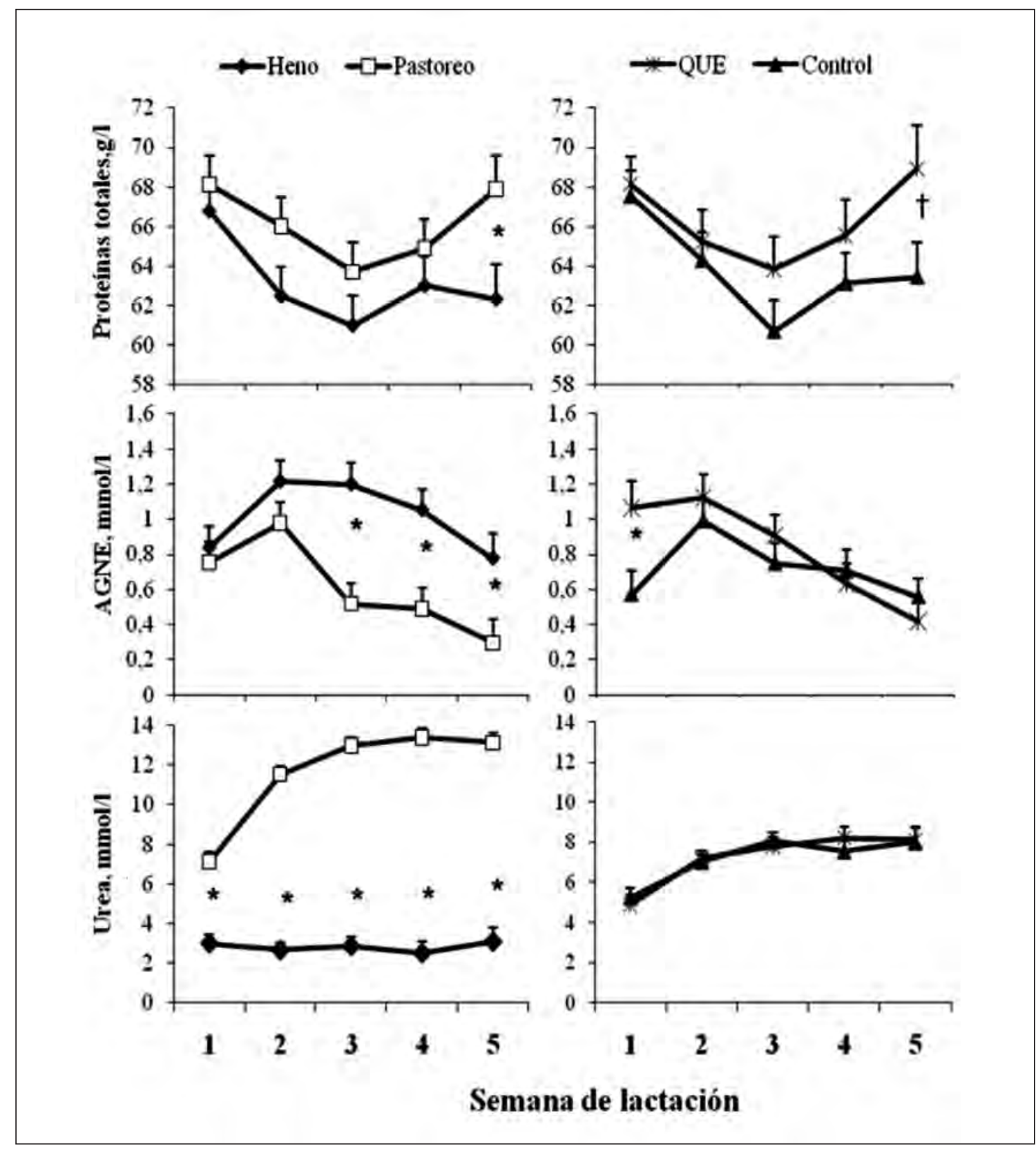

Figura 2. Efecto del tipo de forraje (Heno vs. Pastoreo) y la inclusión de quebracho en el concentrado

(QUE vs. Control) de las dietas de ovejas en lactación sobre los contenidos plasmáticos de proteínas totales, ácidos grasos no esterificados (AGNE) y urea.

Figure 2. Effect of the forage feeding treatment (Hay vs. Pasture) and the inclusion of quebracho in the concentrate (QUE vs. Control) on plasmatic concentrations of total proteins, non-esterified fatty acids and urea during lactation. 


\section{Parámetros productivos de los corderos lechales}

Al igual que en las ovejas, la interacción entre el tipo de forraje y la inclusión de quebracho en el concentrado de las madres no fue significativa en ninguno de los parámetros estudiados en los corderos, por lo que los resultados se presentan por separado para estos efectos.

El peso al nacimiento de los corderos fue similar en todos los lotes, pero los corderos del tratamiento Pastoreo fueron más pesados que los corderos de Heno a partir de la cuarta semana de lactación $(P<0,05)$ (Figura 3$)$. Por ello, los corderos de Pastoreo pesaron más al sacrificio que los de Heno ( $P<0,001$; Tabla 4), y sin diferencia en la edad $(P>0,05)$. Consecuentemente, el tipo de forraje afectó a las ganancias medias de peso durante la lactación (280 vs. 220 g/d, para el tratamiento Pas- toreo y Heno respectivamente; $\mathrm{P}<0,05)$. Las pérdidas de peso debidas al transporte tendieron a ser mayores en los corderos del tratamiento Heno que en los corderos de Pastoreo $(P<0,10)$. El tipo de forraje utilizado en la alimentación de las madres afectó de manera notable a las características de la canal de los corderos lechales (Tabla 4). Paralelamente a los resultados observados en el crecimiento, los corderos de Pastoreo tuvieron mayor PCC y PCF $(\mathrm{P}<0,001)$, y por lo tanto un mayor rendimiento en matadero que los corderos del tratamiento Heno $(P<0,05)$.

La inclusión de quebracho en la dieta de las madres incrementó el peso vivo durante la tercera y cuarta semana $(P<0,05)$ (Figura 3$)$, sin embargo no se observaron diferencias significativas sobre la ganancia media diaria de sus corderos aunque fue un $6,5 \%$ superior en el tratamiento QUE frente al Control, (255 vs. 240; P>0,05). Por ello, los corderos del tra-

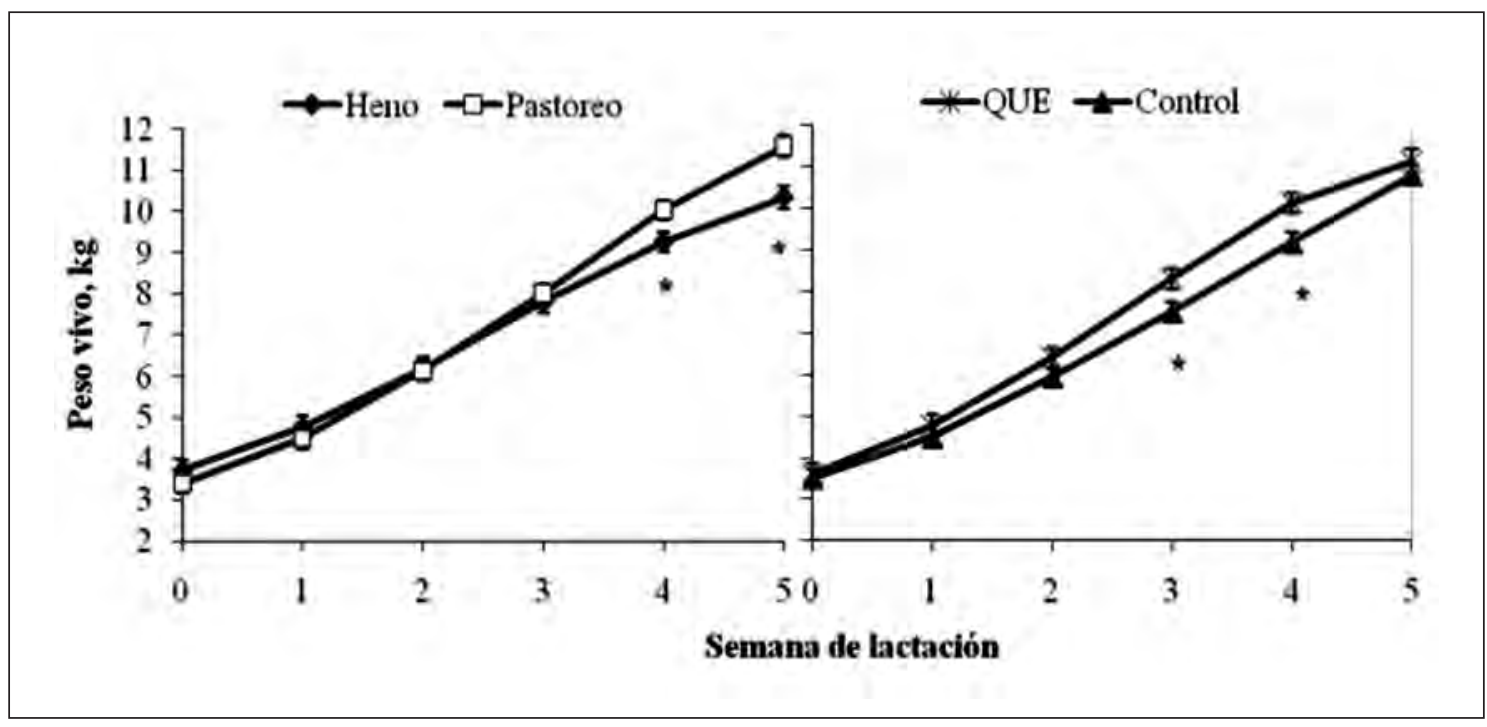

Figura 3. Efecto del tipo de forraje (Heno vs. Pastoreo) y la inclusión de quebracho en el concentrado (QUE vs. Control) de las dietas de ovejas en lactación sobre el peso vivo de los corderos lechales durante la lactación.

Figure 3. Effect of the type of forage (Hay vs. Pasture) and the inclusion of quebracho in the dam s concentrate (QUE vs. Control) on suckling lamb live weight during lactation. 
Tabla 4. Efecto del tipo de forraje (F) y la inclusión de quebracho en el concentrado (C) de la dieta de ovejas sobre los parámetros productivos de sus corderos lechales

Table 4. Effect of the type of forage (F) and the inclusion of quebracho in the concentrate (C) of lactating ewes on their suckling lamb s performance

\begin{tabular}{|c|c|c|c|c|c|c|c|}
\hline & \multicolumn{2}{|c|}{ Forraje } & \multicolumn{2}{|c|}{ Concentrado } & \multirow[b]{2}{*}{$\mathrm{EE}^{2}$} & \multicolumn{2}{|c|}{ Efecto $^{3}$} \\
\hline & Heno & Pastoreo & QUE$^{1}$ & Control & & $\mathrm{F}$ & $\mathrm{C}$ \\
\hline PV Sacrificio, kg & 10,6 & 11,6 & 11,1 & 11,1 & 0,3 & $* * *$ & NS \\
\hline Edad al sacrificio, $d$ & 35,0 & 32,2 & 31,8 & 35,3 & 2,8 & NS & $\dagger$ \\
\hline Pérdidas de PV en transporte, \% & 3,4 & 1,6 & 2,0 & 2,9 & 0,7 & $\dagger$ & NS \\
\hline Peso canal caliente, kg & 6,3 & 7,0 & 6,7 & 6,6 & 0,2 & $* * *$ & NS \\
\hline Peso canal fría, kg & 6,0 & 6,7 & 6,41 & 6,3 & 0,2 & $* * *$ & NS \\
\hline Rendimiento matadero, $\%$ & 56,3 & 58,4 & 58,0 & 56,8 & 0,9 & * & NS \\
\hline Pérdidas de refrigeración, \% & 4,3 & 3,3 & 3,9 & 3,7 & 0,7 & NS & NS \\
\hline
\end{tabular}

${ }^{1}$ QUE: $10 \%$ de quebracho; ${ }^{2}$ Error estándar; ${ }^{3} \dagger=\mathrm{P}<0,10$.

tamiento QUE necesitaron casi 4 días menos que los de Control $(P<0,10)$ para alcanzar similar peso al sacrificio $(P>0,05)$. La inclusión de quebracho en el concentrado $(P>0,05)$ no afectó ni a las pérdidas de peso debido al transporte ni a las características de la canal de los corderos lechales $(P>0,05)$.

\section{Discusión}

\section{Alimentos}

La pradera presentó un valor nutritivo elevado, considerando su alto contenido en PB y bajo contenido en FND, durante toda la lactación de las ovejas. Los partos coincidieron con el inicio del crecimiento del forraje, por lo que al inicio del ensayo la oferta fue escasa pero de alta calidad. Por otra parte, el heno presentó una calidad nutritiva media, con un contenido bajo en PB y alto contenido en FND. La causa de esta diferencia está relacionada con el diferente estado del forraje y el proceso de henificación, que reduce la calidad del forraje (Rotz y Muck, 1994; Dewhurst et al., 2006). Además, la pradera para henificar se cortó cuando la hierba estaba en inicio del estado de maduración, por lo que la calidad fue menor y la cantidad del forraje cosechado mayor. Dado que la producción de forraje de la pradera al inicio del estudio fue muy baja, la carga ganadera aplicada también fue baja, con el fin de garantizar un consumo de forraje suficiente para cubrir las necesidades de los animales. Los resultados mostraron que el pastoreo de la pradera permitió cubrir las necesidades para mantener el PV, la CC y unos niveles normales de metabolitos en sangre de la oveja, así como la producción de leche, que fue comparable a la observada en otros estudios anteriores (Álvarez-Rodríguez et al., 2007), confirmando que la carga ganadera utilizada en el ensayo fue adecuada.

\section{Parámetros productivos de las ovejas}

La evolución del PV, CC y la producción de leche de las ovejas de Heno están de acuerdo con los resultados observados por Joy et al. 
(2012) en un estudio similar de producción de corderos lechales llevado a cabo durante el otoño. Sin embargo, las ovejas en Pastoreo del mencionado estudio presentaron peores rendimientos que las del presente ensayo debido a que no recibieron concentrado como suplemento durante la lactación y fueron similares a los observados por Álvarez-Rodríguez et al. (2007), en el que las ovejas recibieron suplementación de cebada. Los mejores rendimientos observados en las ovejas Pastoreo del presente estudio confirman que la pradera tenía una alta calidad nutritiva, aunque la producción forrajera fuera baja, y permitió cubrir las necesidades de las ovejas autóctonas de formato medio como es la raza Churra Tensina. Además, confirma la recomendación de suplementar a las ovejas durante la lactación realizada por RipollBosch et al. (2014), tanto en las ovejas en pastoreo, para equilibrar la proteína bruta y energía de la dieta, como en las ovejas estabuladas que reciben heno de calidad media para mantener la lactación.

Las diferencias en la producción y calidad de la leche entre las ovejas de Pastoreo y Heno podrían relacionarse con los diferentes contenidos de energía y PB de los forrajes, que fueron un 29 y $71 \%$ mejores en Pastoreo, respectivamente. Está ampliamente aceptado que el estado nutricional y la producción de leche están positivamente relacionados (Caja y Bocquier, 2000).

La mayoría de estudios realizados en razas locales muestran que el pico de producción de leche es al inicio de la lactación (entre las semanas 1 y 3) (Atti et al., 2006; Joy et al., 2008a; Ripoll-Bosch et al., 2014), tal y como se ha registrado en el tratamiento de Heno. Sin embargo, las ovejas de Pastoreo presentaron una evolución diferente, que podría estar relacionada con la cantidad disponible de forraje, baja al inicio de la lactación pero incrementándose a medida que la primavera avanzaba, aumentando de esta manera la energía y proteína ingerida. También puede estar relacionada con el posible incremento de la humedad, ya que se trata de una pradera en crecimiento. La producción de leche media de las ovejas en Pastoreo en el presente estudio fue mayor que la observada por Joy et al. (2012) (1,32 vs. 1,11 kg/día). Tal y como se ha explicado anteriormente, esta diferencia podría deberse a un mayor consumo de energía, debido a la mejor calidad de la pradera, además de a la suplementación de $300 \mathrm{~g}$ de concentrado que se ofreció a las ovejas en el presente estudio.

El alto contenido de PB que presentó la pradera estuvo relacionado con el aumento de la concentración de proteína total y de urea en plasma de las ovejas en Pastoreo, de acuerdo con otros estudios (Jaime y Purroy, 1995; Joy et al., 2008a). La concentración plasmática de urea en ambos tratamientos reflejó diferencias en la ingestión de PB. Las ovejas Pastoreo presentaron un aumento de la urea plasmática entre la primera y tercera semana de lactación, paralelo a la mayor producción forrajera de la pradera, y por tanto a un mayor consumo. Estos resultados están de acuerdo con Fernández et al. (2012), que observaron una mayor concentración de urea en moruecos castrados cuando la dieta presentaba un mayor valor proteico (22\% PB). Por otro lado, la concentración de urea plasmática fue constante en ovejas Heno como consecuencia del contenido constante en PB del heno. Las diferencias en la concentración de AGNE en el plasma estarían relacionadas con las diferencias en el contenido en EM de las dietas (Pastoreo vs. Heno). En este sentido, Jaime y Purroy (1995) observaron que la concentración de AGNE fue mayor en ovejas alimentadas al $70 \%$ de las necesidades energéticas que en aquellas alimentadas al $80 \%$. En el presente estudio, la concentración de AGNE en las ovejas Pastoreo durante la lactación fue inferior a 1,0 mM, umbral que indica un estado catabólico (Russel, 1984), pero en las ovejas Heno 
fue superior a dicho umbral entre las semanas 2 y 4 . Por lo tanto, las ovejas Heno no recibieron suficiente EM para la producción de leche, teniendo que movilizar reservas corporales, como confirmó la disminución de la CC observada en ese tratamiento. La evolución de la concentración de AGNE en plasma a lo largo de la lactación fue inversa a la observada para la CC, de acuerdo con Álvarez-Rodríguez et al. (2012) y Joy et al. (2014).

La inclusión de quebracho en el concentrado no tuvo apenas efectos sobre los rendimientos de las ovejas. En este mismo sentido, Hervás et al. (2003) observaron que cuando las ovejas recibían 0,28 o $83 \mathrm{~g}$ de quebracho/kg mantenían el PV, mientras que cuando recibieron $166 \mathrm{~g} / \mathrm{kg}$ presentaron una pérdida rápida de PV. Toral et al. (2011) observaron que la inclusión de un extracto compuesto por quebracho y castaño (taninos hidrolizables) en $10 \mathrm{~g} / \mathrm{kg}$ de MS no tuvo efecto en la evolución del PV durante la lactación de la oveja. El efecto poco claro de la inclusión de quebracho en la producción y calidad de leche está en parte de acuerdo con los resultados observados cuando el quebracho se incluyó en un 2\% (Toral et al., 2013) o 5,3\% (Buccioni et al., 2015). En el presente estudio, se incluyó un $10 \%$ de quebracho en el concentrado y no afectó a la producción de leche ni al contenido en grasa pero redujo el contenido de proteína. Estudios previos destacan las interacciones entre la semana de lactación y la producción de leche y proteína. Wang et al. (1996), estudiando el efecto del loto (Lotus Corniculatus), un forraje con alto contenido en TC, en la dieta de ovejas, observaron que la producción de leche y proteína fue similar durante las primeras 5 semanas de lactación, pero aumentaron ambas producciones entre las semanas $6^{a}$ y $11^{a}$. Por lo tanto, parece que dependiendo de la semana de lactación la producción y la calidad podrían estar afectadas de manera diferente por el tipo de TC incluido en la dieta.
De manera similar que en la producción de leche, los estudios reflejan un efecto poco claro del quebracho en los metabolitos en sangre. Hervás et al. (2003) no encontraron efectos utilizando las dosis de quebracho de 0, 28 o $83 \mathrm{~g} / \mathrm{kg}$ en la dieta durante 21 días. Otros estudios han descrito una interacción entre la inclusión de quebracho en la dieta y la fase de lactación de la oveja en el contenido en proteína total (Buccioni et al., 2015) y nitrógeno ureico (Fernández et al., 2012). Respecto a otras fuentes de TC, el loto disminuyó la concentración de urea pero no afectó al contenido de AGNE (Wang et al., 1996). En el presente estudio se puede concluir que la cantidad de quebracho añadido en la dieta fue bajo para afectar al PV, CC y la producción y calidad de la leche, siendo razonable la ausencia de efecto del quebracho en las concentraciones de metabolitos del plasma.

\section{Parámetros productivos de los corderos lechales}

El efecto del tipo de forraje sobre el crecimiento de los corderos lechales se debió a las diferencias observadas en la producción de leche de las madres, como se ha indicado previamente (Joy et al., 2012). El mayor crecimiento de los corderos del tratamiento Pastoreo relacionado con su mayor producción de leche fue debido a que un incremento de la energía en la dieta de la madre implica una mayor ingestión de energía y proteína del cordero (Galvani et al., 2014).

El transporte de los corderos al matadero siempre implica pérdidas. En el presente estudio, las pérdidas de los corderos Pastoreo fueron menores a las observadas por Joy et al. (2008b) en corderos ligeros tipo Ternasco (22-24 kg) criados en condiciones similares. La principal causa de esta diferencia pudo ser el menor desarrollo del tracto digestivo en los corderos lechales. Los corderos lechales se alimentan casi exclusivamente de leche, por 
lo que el tracto digestivo debería estar igualmente desarrollado en los tratamientos del presente estudio.

El efecto del tipo de forraje sobre los pesos de la canal y su rendimiento estaría relacionado con el mayor peso al sacrificio en el lote Pastoreo, ya que el PV al sacrificio está correlacionado positivamente con el peso de la canal (Díaz et al. 2003) y el rendimiento canal (Velasco et al. 2000).

En una revisión, Patra y Saxena (2011) concluyeron que la inclusión de concentraciones moderadas de taninos condensados en la dieta puede mejorar el peso corporal, la producción de leche y los parámetros reproductivos de las ovejas, pero no de manera consistente. Sin embargo, en el presente estudio las ganancias medias de los corderos no fueron diferentes significativamente, aunque el ligero incremento debido a la inclusión de quebracho en el concentrado de sus madres podría ser resultado de la tendencia de las ovejas QUE a presentar una mejor producción de leche estándar. Las discrepancias entre los distintos estudios se podrían atribuir parcialmente a la forma en que interaccionan los taninos condensados y las proteínas.

En el presente estudio, la inclusión de quebracho en la dieta de ovejas no afectó a las características de la canal de corderos lechales. De manera similar, Francisco et al. (2015) observaron que los TC del Cistus ladanifer aportados durante 6 semanas en corderos Merino Branco sacrificados con $32-33 \mathrm{~kg}$ no tuvieron efecto en las características de la canal. Sin embargo, las canales de corderos alimentados durante 103-145 días con ensilados con forrajes ricos en TC (Onobrychis viciifolia y Lotus corniculatus) fueron más ligeras y presentaron menor rendimientos que las de los corderos alimentados con ensilados con forrajes sin TC (Medicago sativa y Trifolium pratense) (Girard et al., 2016). El peso al sacrificio fue de $28-29 \mathrm{~kg}$ para los primeros y 34-35 kg para los segundos, por lo que las di- ferencias observadas podrían deberse en parte al diferente peso al sacrificio. Por lo tanto, se necesitan más estudios para comprobar si la inclusión de TC en la dieta tanto de madres como de corderos afecta a las características de la canal cuando los corderos son sacrificados con peso similar.

\section{Conclusiones}

En nuestras condiciones de trabajo, las ovejas en Pastoreo presentaron mayor producción lechera, corderos lechales más pesados al sacrificio y con mayor rendimiento canal que las madres que consumieron Heno. Por tanto, la práctica habitual de estabular a las ovejas al parto y alimentarlas con heno de pradera suplementado con pienso quizá no sea el manejo más adecuado. Una alternativa recomendable sería el pastoreo de ovejas lactantes con suplementación, siempre que se disponga de pasto de buena calidad. La inclusión de quebracho en la dieta de las ovejas lactantes redujo el contenido proteico de la leche, pero tendió a aumentar la producción de leche estándar y mejoró el ritmo de crecimiento de sus corderos, que tendieron a alcanzar el peso de sacrificio 3,5 días antes que el resto de corderos. Debido a la falta de un efecto consistente de la inclusión del quebracho, será interesante analizar en futuros trabajos cómo afecta la inclusión de distintas dosis de taninos condensados, tanto en la dieta de las madres como en la de las crías, a los rendimientos del conjunto oveja-cordero.

\section{Agradecimientos}

Los autores desean agradecer al personal de la Finca Experimental de la Garcipollera y al personal del CITA de Aragón por su ayuda en el desarrollo de la experiencia. En especial deseamos agradecer a J. Casáus y J. Ferrer su 
ayuda en el manejo de los animales, a F. Molino, M.A. Legua y P. Eseverri su trabajo en el laboratorio. Este estudio ha sido financiado por el Instituto Nacional de Investigación y Tecnología Agraria y Alimentaria (INIA RTA2012-080-00, INIA RZP2009-00005 y INIA RZP2013-00001-00) y por las ayudas de los fondos para los grupos consolidados del Gobierno de Aragón (A49, A11). M. Blanco posee un contrato financiado por INIA-FEDER y S. Lobón por una beca predoctoral del Gobierno de Aragón.

\section{Bibliografía}

Álvarez-Rodríguez J, Sanz A, Delfa R, Revilla R, Joy $M$ (2007). Performance and grazing behaviour of Churra Tensina sheep stocked under different management systems during lactation on Spanish mountain pastures. Livestock Science 107: 152-161.

Álvarez-Rodríguez J, Estopañan G, Sanz A, Dervishi E, Govoni N, Tamanini C, Joy M (2012). Carry-over effects of body condition in the early pregnant ewe on peri-partum adipose tissue metabolism. Journal of Animal Physiology and Animal Nutrition 96: 985-992.

AOAC (1999). Official methods of analysis, AOAC International, Maryland, U.S.A.

Atti N, Rouissi H, Othmane MH (2006). Milk production, milk fatty acid composition and conjugated linoleic acid (CLA) content in dairy ewes raised on feedlot or grazing pasture. Livestock Science 104: 121-127.

Bocquier F, Barillet F, Guillouet P, Jacquin M (1993). Prediction of energetic content of ewes milk from different Chemicals-analysis-proposal for a standard milk for Dairy ewes. Annales de Zootechnie 42: 57-66.

Buccioni A, Pauselli M, Viti C, Minieri S, Pallara G, Roscini V, Rapaccini S, Marinucci MT, Lupi P, Conte G, Mele M (2015). Milk fatty acid composition, rumen microbial population, and animal performances in response to diets rich in linoleic acid supplemented with chestnut or quebracho tannins in dairy ewes. Journal of Dairy Science 98: 1145-1156.

Caja G, Bocquier F. (2000). Effects of nutrition on the composition of sheep's milk. En: Cahiers Options Méditerranées (52): Sheep and goat nutrition: Intake, digestion, quality of products and rangelands (Eds. Ledin I. \& Morand-Fehr P.). pp 59-74. Zaragoza:CIHEAM.

Dewhurst RJ, Shingfield KJ, Lee MRF, Scollan ND (2006). Increasing the concentrations of beneficial polyunsaturated fatty acids in milk produced by dairy cows in high-forage systems. Animal Feed Science and Technology 131: 168-206

Díaz MT, Velasco S, Pérez C, Lauzurica S, Huidobro F, Cañeque V (2003). Physico-chemical characteristics of carcass and meat Manchego-breed suckling lambs slaughtered at different weights. Meat Science 65: 1085-1093.

Donney JM, Peart JN, Smith WF, Louda F (1979). A consideration of the techniques for estimation of milk-yield by suckled sheep and comparison of stimates obtained by 2 methods in relation to the effect of breed, level of production and stage of lactation. The Journal of Agricultural Science 92: 123-132.

Fernández HT, Catanese F, Puthod G, Distel RA, ViIlalba JJ. (2012). Depression of rumen ammonia and blood urea by quebracho tannin-containing supplements fed after high-nitrogen diets with no evidence of self-regulation of tannin intake by sheep. Small Ruminant Research, 105: 126-134.

Francisco A, Dentinho MT, Alves SP, Portugal PV, Fernandes F, Sengo S, Jerónimo E, Oliveira MA, Costa P, Sequeira A, Bessa RJB, Santos-Silva J (2015). Growth performance, carcass and meat quality of lambs supplemented with increasing levels of a tanniferous bush (Cistus ladanifer L.) and vegetable oils. Meat Science 100: 275-282.

Galvani DB, Pires CC, Hübner CH, Carvalho S, Wommer TP (2014). Growth performance and carcass traits of early-weaned lambs as affected by the nutritional regimen of lactating ews. Small Ruminant Research 120: 1-5.

Girard M, Dohme-Meier F, Silacci P, Ampuero Kragten S, Kreuzer M, Bee G (2016). Forage legumes rich in condensed tannins may increase $n-3$ 
fatty acid levels and sensory quality of lamb meat. Journal of the Science of Food and agriculture 96: 1923-1933.

Grabber JH, Zeller WE, Mueller-Harvey I (2013). Acetone enhances the direct analysis of procyanidin- and prodelphinidin-based condensed tannins in lotus species by the butanol-HCl-iron assay. Journal of Agricultural and Food Chemistry 61: 2669-2678.

Hervás G, Pérez V, Giráldez FJ, Mantecón AR, Almar MM, Frutos $P$ (2003). Intoxication of sheep with quebracho tannin extract. Journal of Comparative Pathology 129: 44-54.

Jaime C, Purroy A (1995). Level and quality of protein in rations for lactating ewes. Annales De Zootechnie 44: 135-142.

Joy M, Álvarez-Rodríguez J, Revilla R, Delfa R, Ripoll G (2008a). Ewe metabolic performance and lamb carcass traits in pasture and concentrate-based production systems in Churra Tensina breed. Small Ruminant Research 75: 24-35.

Joy M, Ripoll G, Delfa R (2008b). Effects of feeding system on carcass and non-carcass composition of Churra Tensina light lambs. Small Ruminant Research 78: 123-133.

Joy M, Sanz A, Ripoll G, Panea B, Ripoll-Bosch R, Blasco I, Álvarez-Rodríguez J (2012). Does forage type (grazing vs. hay) fed to ewes before and after lambing affect suckling lambs performance, meat quality and consumer purchase intention? Small Ruminant Research 104: 1-9.

Joy M, Ripoll-Bosch R, Sanz A, Molino F, Blasco I, Álvarez-Rodríguez J (2014). Effects of concentrate supplementation on forage intake, metabolic profile and milk fatty acid composition of unselected ewes raising lambs. Animal Feed Science and Technology 187: 19-29.

Mertens DR (1983). Using neutral detergent fiber to formulate dairy rations and estimate the energy content forages. Cornell Nut. Conf. Feed Manuf. Syracuse, NY, p. 60.

Min BR, Barry TN, Attwood GT, McNabb WC (2003). The effect of condensed tannins on the nutrition and health of ruminants fed fresh temperate forages: A review. Animal Feed Science and Technology 106: 3-19.
Montossi F, Font-i-Furnols M, del Campo M, San Julián R, Brito G, Sañudo C (2013). Sustainable sheep production and consumer preference trends: Compatibilities, contradictions, and unresolved dilemmas. Meat Science 95: 772-789.

Moñino I, Martínez C, Sotomayor JA, Lafuente A, Jordán MJ (2008). Polyphenols transmission to Segureño lamb meat from ewes' diet supplemented with the distillate from rosemary (Rosmarinus officinalis) leaves. Journal of Agricultural and Food Chemistry 56: 3363-3367.

Patra AK, Saxena J (2011). Exploitation of dietary tannins to improve rumen metabolism and ruminant nutrition. Journal of the Science of Food and Agriculture 91: 24-37.

Ripoll-Bosch R, Joy M, Sanz A, Blasco I, Ripoll G, Álvarez-Rodríguez J (2014). Effect of concentrate supplementation and prolificacy on the productive and economic performance of autochthonous sheep breeds fed forage-based diets. Spanish Journal of Agricultural Research 12: 1099-1104.

Rotz CA, Muck RE (1994). Changes in Forage Quality During Harvest and Storage. En: Forage Quality, Evaluation, and Utilization (Ed. Fahey, G.C.), pp. 828-868. American Society of Agronomy, Crop Science Society of America, Soil Science Society of America, Madison, WI, EE. UU.

Russel AJF, Doney JM, Gunn RG (1969). Subjective assessment of body fat in live sheep. The Journal of Agricultural Science 72: 451-454.

Russel AJF (1984). Means of assessing the adequacy of nutrition of pregnant ewes. Livestock Production Science 11: 429-436.

Sanz A, Álvarez-Rodríguez J, Cascarosa L, Ripoll G, Carrasco S, Revilla R, Joy M (2008). Características de la canal de los tipos comerciales de cordero lechal, ternasco y pastenco en la raza Churra Tensina. Revista ITEA-Información Técnica Económica Agraria 104: 42-57.

Toral PG, Hervás G, Bichi E, Belenguer Á, Frutos P (2011). Tannins as feed additives to modulate ruminal biohydrogenation: Effects on animal performance, milk fatty acid composition and ruminal fermentation in dairy ewes fed a diet containing sunflower oil. Animal Feed Science and Technology 164: 199-206. 
Toral PG, Hervás G, Belenguer A, Bichi E, Frutos P (2013). Effect of the inclusion of quebracho tannins in a diet rich in linoleic acid on milk fatty acid composition in dairy ewes. Journal of Dairy Science 96: 431-439.

Van Soest PJ, Robertson JB, Lewis BA (1991). Methods for dietary fiber, neutral detergent fiber, and nonstarch polysaccharides in relation to animal nutrition. Journal of Dairy Science 74: 3583-3597.

Velasco S, Lauzurica S, Caneque V, Pérez C, Huidobro F, Manzanares C, Díaz MT (2000). Carcass and meat quality of Talaverana breed sucking lambs in relation to gender and slaughter weight. Animal Science 70: 253-263.
Waghorn G (2008). Beneficial and detrimental effects of dietary condensed tannins for sustainable sheep and goat production-Progress and challenges. Animal Feed Science and Technology 147: 116-139.

Wang Y, Douglas GB, Waghorn GC, Barry TN, Foote AG (1996). Effect of condensed tannins in Lotus corniculatus upon lactation performance in ewes. The Journal of Agricultural Science 126: 353-362.

(Aceptado para publicación el 9 de agosto de 2017) 